\title{
Hybrid PET/MR Imaging in Neurology: Present Applications and Prospects for the Future
}

\author{
Wolf-Dieter Heiss \\ Max Planck Institute for Neurological Research, Cologne, Germany
}

$\mathbf{S}$ (3), PET has developed to multiring systems permitting highresolution and 3-dimensional imaging of various physiologic, functional, and molecular targets. The first applications of PET were in brain research, and despite the many other diagnostic indications, particularly in oncology and cardiology, brain imaging remains a stronghold of PET. Despite the development of multiring systems covering the whole brain, PET images still suffered from limited spatial resolution $(2.3$ and $2.5 \mathrm{~mm}$ in the transaxial and axial directions, respectively, with the High Resolution Research Tomograph (4)), low sensitivity, and insufficient attenuation and scatter correction. Multimodal imaging of physiologic and metabolic variables by PET requires coregistration to CT or MRI for accurate correspondence to the anatomic structures and to pathologic changes. MRI is the best method to image the morphology of the brain in health and disease, and various MR modalities can additionally be used to assess physiologic and metabolic parameters such as vascular supply (contrast-enhanced MRI), perfusion (perfusion-weighted imaging), edema (diffusion-weighted imaging), functional activation (functional MRI), and concentration of defined substrates (MR spectroscopy). Pooling information obtained with MRI and PET has long been performed through a parallel analysis of the sequentially acquired data and, more commonly today, using software coregistration techniques. However, underlying such studies is the assumption that no significant changes in physiologic or cognitive conditions have occurred between the 2 examinations. Although a good assumption for some studies, this may not be the case more generally. For example, a subject's mental state may change on time frames from minutes to even seconds, whereas physiologic and metabolic changes can occur on the order of minutes in some disease conditions such as acute ischemic stroke or migraine. Likewise, rapid changes in baseline physiology can occur with some therapeutic interventions.

One means to address such potential pitfalls is through the simultaneous collection of MRI and PET data. The feasibility of simultaneous PET and MRI data acquisition for human studies was first demonstrated in 2007, and proof-of-principle brain data were collected using a prototype MRI-compatible PET insert-called

Received Mar. 7, 2016; revision accepted Mar. 8, 2016.

For correspondence and reprints contact: Wolf-Dieter Heiss, Max Planck Institute for Neurological Research, Gleueler Strasse 50, Cologne, 50931, Germany.

E-mail: wdh@sf.mpg.de

Published online Apr. 7, 2016.

COPYRIGHT (C) 2016 by the Society of Nuclear Medicine and Molecular Imaging, Inc.

DOI: 10.2967/jnumed.116.175208
BrainPET-positioned inside a commercially available 3-T MRI Trio system (Siemens Medical Solutions) (5). In 2010, a fully integrated PET/MR scanner also became available for human whole-body imaging (Biograph mMR) (6). Simultaneous PET/MR allows spatial and temporal correlation of the signals from both modalities, creating opportunities impossible to realize using sequentially acquired data. The features of this new technology may be particularly appealing to applications for translational research in neuroscience, considering that MRI represents the first-line diagnostic imaging modality for numerous indications and that a great number of specific PET tracers are available today to assess functional and molecular processes in the brain.

Simultaneous imaging certainly yields benefits with regard to patient management and time saving. Avoiding the repositioning of the patient improves coregistration and localization of anatomic structures and lesions: this is of great advantage in the presurgical diagnosis of patients with focal epilepsy, for which small lesions, hypoplasias, or heterotopies can be delineated $(7,8)$. Improved differentiation of different tissue types by combined metabolic and morphologic imaging is of great importance in the differential diagnosis of brain tumors, for grading of gliomas, for the assessment of progression and the distinction between necrosis and recurrence; it also helps in the selection of the most promising place for biopsies and in the evaluation of treatment effects (7,9-14). Further information on effects of tumors on morphology, function, and metabolism of the surrounding brain may be obtained by adding diffusion tensor imaging/fiber tracking, functional MRI, perfusion-weighted imaging, MR spectroscopy, and activation PET to the multimodal imaging (15-17), by which anaerobic changes in energy metabolism in tumor and peritumor tissue, alterations in efferent and connecting fiber tracts, and task-related activation patterns within functional networks can be visualized.

Coregistration of structure and metabolism together with simultaneous assessment of synaptic function are important for early recognition and differential diagnosis of cognitive impairment and for understanding the pathophysiology — for example, deposition of amyloid, tau, or other abnormal proteins - of degenerative disorders. Early diagnosis of Alzheimer dementia and even detection of prestates of this devastating disorder can be achieved by MRI (hippocampal atrophy) combined to PET for measurement of glucose consumption and accumulation of amyloid and tau, which should be used for the selection of patients in treatment trials; the multimodal imaging permits also the differential diagnosis to other degenerative diseases (18-22). Further insights into the development of cognitive disturbances will be obtained by adding PET studies of synaptic function, for example, cholinergic and serotoninergic transmission $(18,23)$.

Synergistic measurement of different physiologic parameters can explain functional impairment and predicts the development of 
irreversible neuronal damage in ischemic stroke and therefore is crucial for therapeutic decisions. Because PET studies of regional cerebral flow and oxygen consumption are time consuming and not feasible for patients with acute ischemic stroke, noninvasive nonquantitative determinations of flow and tissue condition by perfusion-weighted and diffusion-weighted MRI are often used for classification of patients, but for a reliable definition of the mismatch as a measure of the penumbra-a state of critically perfused tissue with maintained morphologic integrity-validation of parameters is necessary, which can be obtained only by comparative studies of PET and MRI (24). With the use of advanced techniques for analysis of MR data, determinations of perfusion by both methods are comparable and may be applied successfully for the description of ischemic compromise $(25,26)$. Simultaneous PET/MR studies will be able to detect anaerobic glycolysis in ischemic tissue and will play a role in recognizing the impact of neuroinflammation on progress of tissue damage as well as on repair mechanisms after ischemia $(27,28)$.

Using the unique capacities of hybrid PET/MR for simultaneous real-time recording of functional, metabolic, physiologic, and morphologic data opens new fields in clinical research: activation studies by PET and functional MRI combined to diffusion tensor imaging permit the plotting of functional networks in health and disease and demonstrate the effect of noninvasive (repetitive transcranial magnetic stimulation, direct current stimulation) and deep brain stimulation (implanted electrodes) $(15,29)$. Tracers for transmitters, receptors, and enzymes further elucidate the involvement of synaptic function in special tasks and uncover changes by diseases and drugs $(30,31)$. Tracers were also developed to identify residual tumor tissue for image-guided vector application and for identification of enzyme expression in glioma cells as a target for selective treatment $(32,33)$.

In the future, translational stem cell research will benefit from innovative applications of PET/MR. Experiments demonstrating the differentiation of stem cells to dopaminergic neurons and their function might be replicated in humans by ${ }^{11} \mathrm{C}-2 \beta-$ carbomethoxy-3 $\beta$-(4-fluoro)tropane PET and perfusion-weighted MRI (34); accumulation of implanted iron-labeled stem cells in border zones of brain tumors (35) and migration of such stem cells to ischemic lesions can be demonstrated (36); and experiments to even detect the mobilization of endogenous neural stem cells and their migration to and proliferation around ischemic lesions ( $3^{\prime}$-deoxy-3'-fluorothymidine PET and MRI of iron oxidelabeled cells) might be established (37). The viability of stem cells can be documented by MRI combined to PET imaging of reporter genes (33). Another interesting field is angiogenesis, which can be investigated by PET of ${ }^{18} \mathrm{~F}$-galacto-RGD and dynamic contrastenhanced MRI, and might be a new target for selective tumor therapy $(38,39)$.

Predominantly clinical applications of hybrid PET/MR benefit from the isocentric and simultaneous measurements warranting perfect anatomic matching. Thereby attenuation correction is facilitated, and prospective and retrospective motion correction is possible. MRI combines good soft-tissue contrast with no additional ionizing radiation and adds further functional data by spectroscopy, functional MRI, and dynamics of contrast medium. For clinical and translational research, hybrid PET/MR opens innovative strategies to improve our insight into the complex function of the brain and to deepen our understanding of the pathophysiology of central nervous system disorders. PET/MR will play a crucial role in the transfer of developing therapeutic concepts from animal experiments to human application.

\section{DISCLOSURE}

No potential conflict of interest relevant to this article was reported.

\section{REFERENCES}

1. Ter-Pogossian MM, Phelps ME, Hoffman EJ, Mullani NA. A positron-emission transaxial tomograph for nuclear imaging (PETT). Radiology. 1975;114:89-98.

2. Yamamoto YL, Thompson CJ, Meyer E, Robertson JS, Feindel W. Dynamic positron emission tomography for study of cerebral hemodynamics in a cross section of the head using positron-emitting ${ }^{68} \mathrm{Ga}-\mathrm{EDTA}$ and ${ }^{77} \mathrm{Kr}$. J Comput Assist Tomogr. 1977;1:43-56.

3. Phelps ME, Hoffman EJ, Huang SC, Kuhl DE. ECAT: a new computerized tomographic imaging system for positron-emitting radiopharmaceuticals. $\mathrm{J}$ Nucl Med. 1978;19:635-647.

4. Wienhard K, Schmand M, Casey ME, et al. The ECAT HRRT: performance and first clinical application of the new high resolution research tomograph. IEEE Trans Nucl Sci. 2002;49:104-110.

5. Schlemmer HP, Pichler BJ, Schmand M, et al. Simultaneous MR/PET imaging of the human brain: feasibility study. Radiology. 2008;248:1028-1035.

6. Drzezga A, Souvatzoglou M, Eiber M, et al. First clinical experience with integrated whole-body PET/MR: comparison to PET/CT in patients with oncologic diagnoses. J Nucl Med. 2012;53:845-855.

7. Catana C, Drzezga A, Heiss WD, Rosen BR. PET/MRI for neurologic applications. J Nucl Med. 2012;53:1916-1925.

8. Shin HW, Jewells V, Sheikh A, et al. Initial experience in hybrid PET-MRI for evaluation of refractory focal onset epilepsy. Seizure. 2015;31:1-4.

9. la Fougère C, Suchorska B, Bartenstein P, Kreth FW, Tonn JC. Molecular imaging of gliomas with PET: opportunities and limitations. Neuro-oncol. 2011;13:806-819.

10. Preuss $\mathrm{M}$, Werner $\mathrm{P}, \mathrm{Barthel} \mathrm{H}$, et al. Integrated PET/MRI for planning navigated biopsies in pediatric brain tumors. Childs Nerv Syst. 2014;30:1399-1403.

11. Burhan AM, Marlatt NM, Palaniyappan L, Anazodo UC, Prato FS. Role of hybrid brain imaging in neuropsychiatric disorders. Diagnostics (Basel). 2015;5: 577-614.

12. Bisdas S, La Fougere C, Ernemann U. Hybrid MR-PET in neuroimaging. Clin Neuroradiol. 2015;25(suppl 2):275-281.

13. Werner P, Barthel H, Drzezga A, Sabri O. Current status and future role of brain PET/MRI in clinical and research settings. Eur J Nucl Med Mol Imaging. 2015;42:512-526.

14. Filss CP, Galldiks N, Stoffels G, et al. Comparison of ${ }^{18}$ F-FET PET and perfusion-weighted MR imaging: a PET/MR imaging hybrid study in patients with brain tumors. J Nucl Med. 2014;55:540-545.

15. Neuner I, Kaffanke JB, Langen KJ, et al. Multimodal imaging utilising integrated MR-PET for human brain tumour assessment. Eur Radiol. 2012;22:2568-2580.

16. Jeong JW, Juhasz C, Mittal S, et al. Multi-modal imaging of tumor cellularity and tryptophan metabolism in human Gliomas. Cancer Imaging. 2015;15:10.

17. Morana G, Piccardo A, Puntoni M, et al. Diagnostic and prognostic value of ${ }^{18} \mathrm{~F}-$ DOPA PET and ${ }^{1} \mathrm{H}-\mathrm{MR}$ spectroscopy in pediatric supratentorial infiltrative gliomas: a comparative study. Neuro-oncol. 2015;17:1637-1647.

18. Drzezga A, Barthel H, Minoshima S, Sabri O. Potential clinical applications of PET/MR imaging in neurodegenerative diseases. J Nucl Med. 2014;55:47S-55S.

19. Hitz S, Habekost C, Furst S, et al. Systematic comparison of the performance of integrated whole-body PET/MR imaging to conventional PET/CT for ${ }^{18} \mathrm{~F}-$ FDG brain imaging in patients examined for suspected dementia. $\mathrm{J} \mathrm{Nucl} \mathrm{Med}$. 2014;55:923-931.

20. Barthel H, Schroeter ML, Hoffmann KT, Sabri O. PET/MR in dementia and other neurodegenerative diseases. Semin Nucl Med. 2015;45:224-233.

21. Tahmasian M, Shao J, Meng C, et al. Based on the network degeneration hypothesis: separating individual patients with different neurodegenerative syndromes in a preliminary hybrid PET/MR study. J Nucl Med. 2016;57:410-415.

22. Harada R, Okamura N, Furumoto S, et al. ${ }^{18} \mathrm{~F}-\mathrm{THK} 5351$ : a novel PET radiotracer for imaging neurofibrillary pathology in Alzheimer disease. J Nucl Med. 2016;57: 208-214.

23. Herholz K, Weisenbach S, Kalbe E, Hilker R, Mielke R, Heiss WD. Dementia in Lewy body disease is related to cholinergic but not to dopaminergic impairment. Neuroimage. 2004;22:T179.

24. Heiss WD, Sobesky J. Comparison of PET and DW/PW-MRI in acute ischemic stroke. Keio J Med. 2008;57:125-131.

25. Zhang K, Herzog H, Mauler J, et al. Comparison of cerebral blood flow acquired by simultaneous $\left[{ }^{15} \mathrm{O}\right]$ water positron emission tomography and arterial spin labeling magnetic resonance imaging. J Cereb Blood Flow Metab. 2014;34:1373-1380. 
26. Werner P, Saur D, Zeisig V, et al. Simultaneous PET/MRI in stroke: a case series. J Cereb Blood Flow Metab. 2015;35:1421-1425.

27. Tsuchida C, Kimura H, Sadato N, Tsuchida T, Tokuriki Y, Yonekura Y. Evaluation of brain metabolism in steno-occlusive carotid artery disease by proton MR spectroscopy: a correlative study with oxygen metabolism by PET. $J$ Nucl Med. 2000;41:1357-1362.

28. Thiel A, Radlinska BA, Paquette C, et al. The temporal dynamics of poststroke neuroinflammation: a longitudinal diffusion tensor imaging-guided PET study with ${ }^{11} \mathrm{C}-\mathrm{PK} 11195$ in acute subcortical stroke. J Nucl Med. 2010;51: 1404-1412.

29. Thiel A, Habedank B, Herholz K, et al. From the left to the right: how the brain compensates progressive loss of language function. Brain Lang. 2006;98: 57-65.

30. Wey HY, Catana C, Hooker JM, et al. Simultaneous fMRI-PET of the opioidergic pain system in human brain. Neuroimage. 2014;102:275-282.

31. Qin P, Wu X, Duncan NW, et al. GABAA receptor deficits predict recovery in patients with disorders of consciousness: a preliminary multimodal $\left[{ }^{11} \mathrm{C}\right]$ flumazenil PET and fMRI study. Hum Brain Mapp. 2015;36:3867-3877.

32. Jacobs A, Voges J, Reszka R, et al. Positron-emission tomography of vectormediated gene expression in gene therapy for gliomas. Lancet. 2001;358:727-729.
33. Chao F, Shen Y, Zhang H, Tian M. Multimodality molecular imaging of stem cells therapy for stroke. Biomed Res Int. 2013;2013:849819.

34. Bjorklund LM, Sanchez-Pernaute R, Chung S, et al. Embryonic stem cells develop into functional dopaminergic neurons after transplantation in a Parkinson rat model. Proc Natl Acad Sci USA. 2002;99:2344-2349.

35. Aboody KS, Brown A, Rainov NG, et al. Neural stem cells display extensive tropism for pathology in adult brain: evidence from intracranial gliomas. Proc Natl Acad Sci USA. 2000;97:12846-12851.

36. Hoehn M, Kustermann E, Blunk J, et al. Monitoring of implanted stem cell migration in vivo: a highly resolved in vivo magnetic resonance imaging investigation of experimental stroke in rat. Proc Natl Acad Sci USA. 2002;99:16267-16272.

37. Rueger MA, Schroeter M. In vivo imaging of endogenous neural stem cells in the adult brain. World J Stem Cells. 2015;7:75-83.

38. Haubner R, Wester HJ, Weber WA, et al. Noninvasive imaging of $\alpha_{\mathrm{v}} \beta_{3}$ integrin expression using ${ }^{18} \mathrm{~F}$-labeled RGD-containing glycopeptide and positron emission tomography. Cancer Res. 2001;61:1781-1785.

39. Beer AJ, Lorenzen S, Metz S, et al. Comparison of integrin $\alpha_{\mathrm{v}} \beta_{3}$ expression and glucose metabolism in primary and metastatic lesions in cancer patients: a PET study using ${ }^{18}$ F-galacto-RGD and ${ }^{18}$ F-FDG. J Nucl Med. 2008;49: $22-29$. 